Annales de Parasitologie (Paris), t. 41, 1966, $\mathrm{n}^{\circ} 5$, pp. 503 à 512

\title{
Sur la présence, chez une femelle de Simulium damnosum Théobald (Diptera, Simuliidae), d'une larve de Perilampidae (Hymenoptera, Cbalcidoidea)
}

\author{
Par Michel LAMONTELLERIE
}

Au cours de recherches sur l'épidémiologie de l'Onchocercose en Haute-Volta, nous avons rencontré une femelle de Simulium damnosum Théobald, 1903, contenant, à l'intérieur du thorax, une larve d'insecte inconnue jusqu'ici, semble-t-il, chez les Simuliidae.

Cet exemplaire parasité a été découvert dans une série de 2.220 femelles de $S$. damnosum disséquées durant la saison des pluies $1962-63$ près du village de Lenga $\left(11^{\circ} 36 \mathrm{~N}-0^{\circ} 46 \mathrm{~W}\right.$, Cercle de Garango).

La femelle de $S$. damnosum en cause a été capturée le 22-10-62, entre 8 et 10 heures ; elle présentait un statut physiologique de femelle pare $\left(\mathrm{N}^{\circ} 68\right.$ des 346 exemplaires pares récoltés le même jour, au même endroit, entre 6 et 18 heures): non gorgée, dépourvue de graisse abdominale, à tubes de Malpighi semi-clairs, présentant à la fois des reliques folliculaires normales et des reliques folliculaires anormales, sans œufs résiduels ; cette femelle de $S$. damnosum était, par ces divers caractères, semblable à de nombreuses autres non parasitées et il ne nous est pas apparu que la présence du parasite dans l'organisme de l'hôte ait influencé l'aspect ou la physiologie de celui-ci, à l'encontre de ce qui se passe lors du parasitisme des femelles de $S$. damnosum par des grégarines ou certaines larves de Nématodes.

Les observations concernant le parasitisme d'adultes de Diptères hématophages par des larves d'autres insectes sont très rares et se rapportent soit à des Strepsiptères (Philip, 1949, chez Chrysops ; Rageau, 1951, chez Phlebotomus), soit à un Hyménoptère (Larivière et Abonnenc, 1958, chez Phlebotomus), c'est pourquoi nous avons jugé utile de décrire ici la larve que nous avons récoltée. 


\section{Description}

Larve apode, de 0,19 $\mathrm{mm}$ de long sur 0,09 $\mathrm{mm}$ de largeur maximale (au niveau des segments 4-5 après la tête), de forme ovale allongée, aplatie dorsoventralement, à face dorsale légèrement bombée, de coloration générale brun-roux.

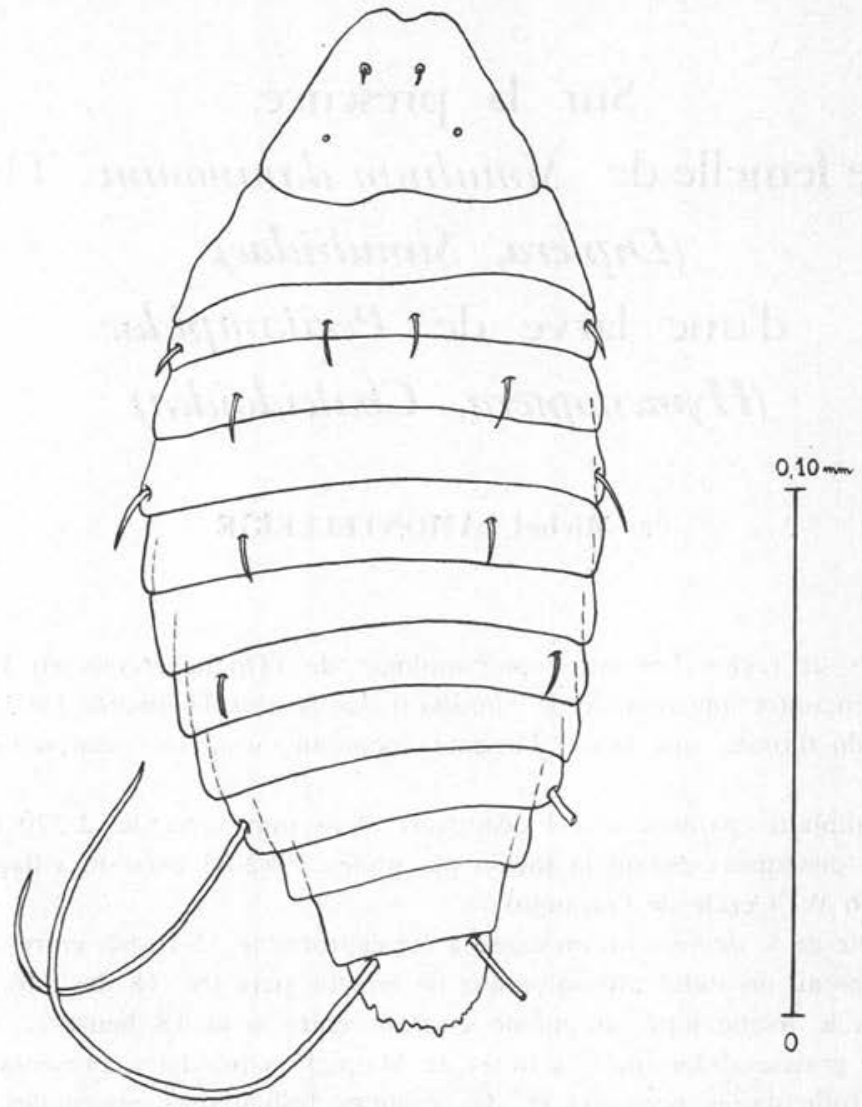

Fig. 1. - Face dorsale

La longueur totale de l'insecte, à l'état vivant, devait être probablement légèrement supérieure, certains segments du corps sont, en effet, plus ou moins télescopés les uns dans les autres, ce qui pourrait marquer une rétraction longitudinale.

Le corps comprend une tête bien individualisée et 12 segments distincts dont les onze premiers sont fortement sclérotisés: le tégument de la face dorsale forme des plaques se prolongeant latéralement jusqu'à la région ventrale et chaque segment du corps (y compris la tête et à l'exception du segment XII) apparaît comme une sorte d'anneau rigide ouvert à la face ventrale. Les divers segments sont séparés par des membranes souples et la région médioventrale est molle et un peu moins pigmentée. 
Tête : Cordiforme en vue dorsale, encochée aux bords antérieur et postérieur, plus large que longue. Présente deux courtes soies pointues insérées dans une fossette (antennes ?) et, en arrière de celles-ci, latéralement, deux petites fossettes arrondies (organes sensoriels ?).

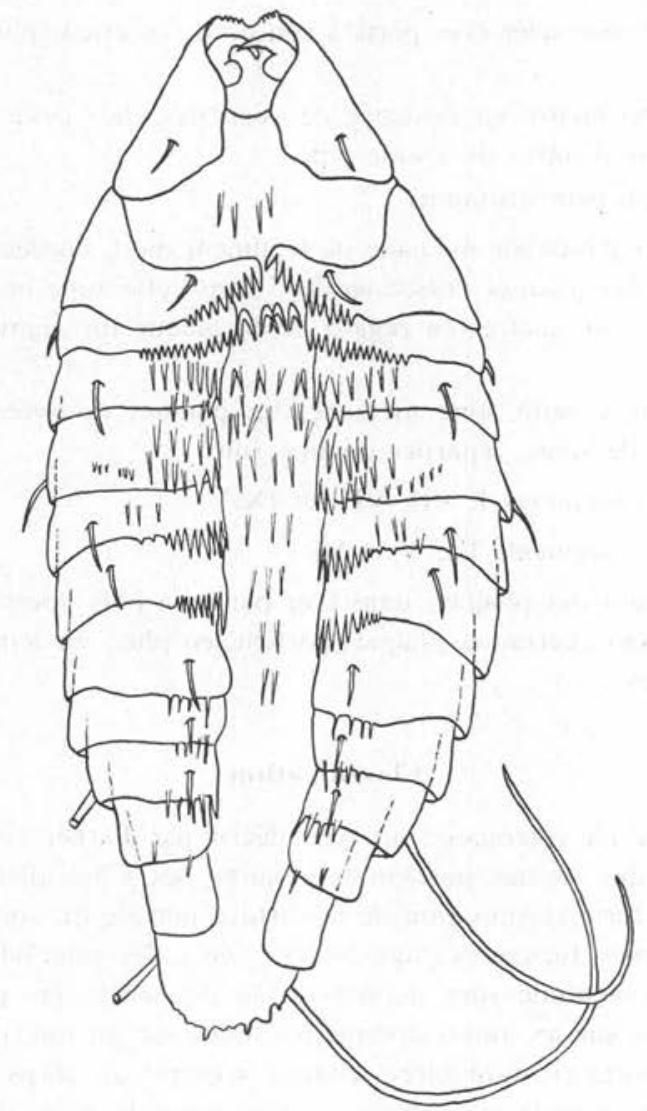

FIG. 2. - Face ventrale

La face ventrale présente, à la région antérieure, une sorte d'entonnoir bordé par le tégument dur, dans lequel sont situées deux fortes mandibules en virgule, très acérées à l'extrémité. En avant des mandibu'es, présence de cinq paires de très fins organes digités (organes sensoriels ?). Latéralement à cet entonnoir buccal, le tégument porte, de chaque côté, une assez forte soie. 
Corps : Dorsalement, porte un certain nombre de soies réparties sur différents segments, à raison de deux ou quatre par segment :

- Deux paires sur le segment II.

- Une seule paire sur les segments III, IV, V, VII, IX et XII. Les soies des segments IX et XII sont très déve'oppées, en particulier celles du segment XII (sur notre exemplaire, les soies droites de ces deux segments sont sectionnées).

Le segment XII, non sclérotisé, porte à son bord postérieur plusieurs petites expansions digitiformes.

Nous n'avons pu mettre en évidence de stigmates chez cette larve, probablement propneustique comme d'autres du même type.

Ventralement, on peut distinguer :

- Une zone longitudinale médiane de tégument mou, bordée latéralement par les extrémités ventrales des plaques dorsolatérales; dans cette zone on note la présence de nombreuses épines, dont quatre (en regard de la plaque du segment II) en forme de crochets bidentés.

- Latéralement à cette zone médiane, les plaques dorsoventrales portent deux séries longitudinales de soies, réparties comme suit:

* Série interne : segments I, VII, VIII et IX.

* Série externe : segments III, V et VI.

Le bord postérieur des plaques, dans leur partie la p'us interne, est armé de nombreuses et fortes épines ; certaines plaques portent, en plus, sur leur surface, des épines diversement disposées.

\section{Classification}

La larve décrite ici correspond au type décrit par Parker (1924) dans les larves du « groupe VII » des formes post-embryonnaires des Chalcidiens... «Ces larves se caractérisent par la forte chitinisation de la surface dorsale du corps qui est composée d'une série de plaques fortement pigmentées; seu's, les intervalles qui séparent les segments et la face ventrale sont dépourvus de pigments; les plaques segmentaires s'imbriquent les unes sur les autres comme les tuiles sur un toit, de sorte que la larve ressemble à un cloporte en miniature... chaque segment du corps porte des soies sensorielles sur les faces dorsa!e et ventrale; la face ventrale a en outre de fortes épines locomotrices... ».

Le type de ces larves a été dénommé «planidium» par H. S. Smith, en 1912 ; le premier exemplaire décrit l'a été par Wheeler (1907), chez une fourmi du Sud-Ouest des Etats-Unis.

De même, Clausen (1940), puis Berland (1951), donnent de la larve planidium une description correspondant à celle de notre exemplaire... « corps fusiforme, avec sclérites segmentaires durs, souvent avec de fortes épines, abdomen se terminant par un disque adhésif...». Nous pensons que, chez notre larve, les expansions que nous 
avons signalées au bord postérieur du segment XII représentent le disque adhésif plus ou moins rétracté.

Les larves de ce type sont des larves de stade I et appartiennent à deux familles d'Hyménoptères Chalcidoidea, les Eucharidae et les Perilampidae. L'étude de diverses descriptions de planidia faites par plusieurs auteurs (Smith, 1912; Thompson, 1915 ; Ford, 1922 ; Parker, 1924 ; Bergold et coll., 1937 ; Clausen, 1940 ; Clancy, 1946; Principi, 1947 ; Tripp, 1962 ; Farooqi et coll., 1965...) nous amène à la conclusion que notre exemplaire appartient à la Famille des Perilampidae.

En conclusion : le parasite décrit ici est une larve primaire (stade I) d'Hyménoptère, de type planidium, appartenant à la Super-Famille des Chalcidoidea et à la Famille des Perilampidae, dont il n'existe, à notre connaissance, qu'un Genre, Perilampus.

\section{Biologie des Perilampidae}

Généralités : Le Genre Perilampus comprend des formes généralement hyperparasites de Lépidoptères ou d'Hyménoptères Symphytes, à travers des Diptères (Tachinidae et Sarcophagidae) ou des Hyménoptères Térébrants (Ichneumonidae et Braconidae). Il est aussi fréquemment hyperparasite d'autres insectes, en particulier des Hémiptéroïdes, à travers des Tachinidae: Dupuis (1963), cite à ce sujet, plusieurs auteurs ayant obtenu des Perilampidae à partir de Phasiinae (Tachinidae) provenant d'Hétéroptères ; il rapporte en outre une récolte personnelle d'un planidium de Perilampus chez un Tachinidae parasite de l'Hémiptère Ceresa bubalus.

Cependant, sur ce schéma général, existent plusieurs modalités indiquant nettement la plasticité parasitaire du Genre :

- En effet, d'une part, certains Perilampus peuvent être, chez un même Insecte, soit parasites primaires, soit hyperparasites; ainsi Tripp (1962) a fait une étude sur $P$. hyalinus Say, parasite primaire de l'Hyménoptère Diprionidae Neodiprion swainei Midd. ou hyperparasite de la même espèce à travers un Diptère Tachinidae (Spathimeigenia spinigera Tnsd.) ou des Hyménoptères Ichneumonidae (Olesicampe lophyri Riley et Endasys subclavatus Say).

- D'autre part, certains Perilampus sont uniquement parasites primaires, semble-t-il, de certains insectes :

* Planipennes: Le Genre Chrysopa est assez fréquemment parasité par plusieurs espèces ( $P$. chrysopae Cwfd, $P$. laevifrons Dalm.), comme l'ont montré en particulier Clancy (1946), Principi (1947) et Smirnoff (1957).

* Lépidoptères : Rondani (1872 et 1876) a signalé $P$. laevifrons comme parasite primaire de deux Tortricidae, Carpocapsa pomonella L. et Evetria buolina Schiff.

* Hyménoptères Symphytes : Martelli (1932) a signalé P. italicus F. chez Athalia colibri Christ. (= A. spinarum F.).

* Coléoptères: De Gaulle (1908) a signalé la présence de P. laevifrons chez un Cerambycidae, Exocentrus punctipennis Muls. 
- Enfin, un certain nombre de planidia ont été décrits, ou des adultes de Perilampus ont été obtenus de plusieurs insectes sans que leur degré de parasitisme ait été défini :

* Orthoptères : Ford (1922) a décrit un planidium chez Conocephalus fasciatus (De Geer). Smith C. W. (1939) et Smith R.W. (1958) citent un certain nombre d'Orthoptères parasités par des Perilampus.

* Coléoptères : Berland (1940) signale un Perilampus obtenu d'un Hylophilidae.

- Signalons que Thompson (1915) a décrit un planidium de Perilampidae chez un Myriapode Chilopode, Lithobius forficatus L., celui-ci serait hyperparasite, à travers un Diptère Tachinidae.

\section{ESPÈCES DONT LA BIOLOGIE EST EN PARTIE CONNUE :}

* P. chrysopae Cwfd: parasite primaire de Planipennes.

* $P$. hyalinus Say: hyperparasite ou parasite primaire d'un Hyménoptère Symphyte (Tripp, 1962) ; Hyperparasite de Lépidoptères (Smith, 1912).

* P. italicus F.: parasite primaire d'un Hyménoptère Symphyte, Athalia colibri (Martelli, 1932).

* P. laevifrons Dalm. : Parasite primaire de Planipennes (Principi, 1947 ; Smirnoff, 1957), de Lépidoptères (Rondani, 1872 et 1876) et d'un Coléoptère (De Gaulle, 1908).

* P. microgastris Ferrière: Hyperparasite d'un Lépidoptère Pyralididae (Farooqi et coll., 1965).

* P. tristis Mayr: Hyperparasite de Lépidoptères, à travers des Tachinidae, Ichneumonidae et Braconidae (Bergold et Ripper, 1937).

\section{Evolution :}

La femelle de Perilampus pond de 200 à 500 œufs, jamais directement sur l'hôte, mais sur les végétaux.

La larve primaire, ou planidium, agile et mobile, au passage d'un hôte éventuel, s'attache à celui-ci puis pénètre dans sa cavité générale.

En cas de parasitisme primaire, le planidium se déplace dans le corps de son hôte, sans prendre de nourriture, jusqu'à la nymphose de celui-ci. A la nymphose de l'hôte, le planidium traverse la cuticule de ce dernier et se conduit alors en ectoparasite: il se nourrit, se développe et mue ; le type morphologique planidium disparaît alors et les larves successives se rapprochent, morphologiquement, du type larvaire classique des Hyménoptères. La vie larvaire comporterait 4 stades (Parker, 1924).

En cas d'hyperparasitisme, le planidium se déplace à l'intérieur du corps de l'hôte primaire, puis pénètre dans l'hôte secondaire. Au moment où l'hôte secondaire sort de l'hôte primaire et se nymphose, le planidium devient externe à la nymphe ; il se développe alors comme dans le cas précédent. 
Il est remarquable que le planidium se fixe sur l'hôte primaire et pénètre dans sa cavité générale même si ce dernier n'est pas encore parasité lui-même. Il a été démontré que le planidium se fixe sur tout objet en mouvement, ce qui explique, d'une part, l'absence de spécificité de plusieurs Perilampus et, d'autre part, qu'on ait cité ou décrit un certain nombre de planidia sans pouvoir définir leur évolution ultérieure.

\section{Planidia de Diptères hématophages}

Larivière et Abonnenc (1958) ont décrit un parasite de Phlebotomus freetownensis var. sudanicus Théodor, 1933 qui est un planidium de Perilampus. Il semble que ce planidium, fixé au clypéus de son hôte, doit être interprété comme étant fixé depuis peu et n'ayant pas encore pénétré dans le corps de l'hôte. Ce parasite présente un certain nombre d'analogies avec celui que nous avons décrit, bien qu'il appartienne, indiscutablement, à une espèce différente. C'est là, à notre connaissance, le premier cas de planidium de Perilampidae décrit chez un Diptère hématophage.

En ce qui concerne notre larve, il est connu que les Simuliidae, comme les autres Diptères hématophages, se nourrissent de sucs végétaux. Nous devons donc admettre que notre planidium s'est fixé sur la Simulie au moment où celle-ci se nourrissait sur un végétal, la pénétration dans le corps de l'hôte n'a eu lieu que plus tard, à un moment impossible à fixer, la Simulie considérée étant pare et relativement âgée.

Etant donné que l'on ne connaît rien, ni sur la systématique, ni sur la biologie des Perilampidae de la Région éthiopienne, il n'est pas possible à l'heure actuelle de rattacher les deux planidia connus des Diptères hématophages à des espèces données.

Ce qu'on peut cependant affirmer, c'est que, dans les deux cas, il s'agit d'un parasitisme accidentel, véritable impasse parasitaire. Nous sommes même là à la limite du parasitisme réel car il ne semble pas que les hôtes aient eu à subir un quelconque préjudice dû à la présence de la larve primaire du Perilampidae.

Qu'il nous soit permis de remercier ici tout particulièrement MM. Benard et Dupuis, du Muséum National d'Histoire Naturelle, pour l'aide efficace qu'ils nous ont apportée au cours de l'étude de ce parasite et au cours de nos recherches bibliographiques.

\section{Résumé}

Description d'une larve de type planidium d'Hyménoptère Chalcidoidea, Perilampidae, parasite accidentel d'une femelle de Simulium damnosum.

L'auteur met à profit cette description pour faire une brève revue de la biologie des Perilampidae. 


\section{Bibliographie}

Bergold (G.) et Ripper (W.), 1937. - Perilampus tristis Mayr als Hyperparasit des Kieferntreibwicklers (Rhyiacionia buolina Schift). Zeitschr. für Parasitenkunde, 9, 394-417.

Berland (L.), 1940. - Hyménoptères, in La Faune de la France de Rémy Perrier, 7.

—, 1951. - Hyménoptères, formes larvaires, in Grassé, traité de Zoologie, 10, 846.

ClanCY (D. W.), 1946. - The insect parasites of the Chrysopidae (Neuroptera). Univ. Calif. Publ. Ent., 7, 403-496.

Clausen (C. P.), 1940. - Entomophagous Insects. Mc Graw-Hill Book C ${ }^{\circ}$ Inc., New York, $688 \mathrm{p}$.

DE Gaulle (J.), 1908. - Catalogue systématique et biologique des Hyménoptères de France. Feuille Jeunes Nat., 1-171, Paris.

DupuIs (Cl.), 1963. - Essai monographique sur les Phasiinae (Diptères Tachinaires parasites d'Hétéroptères). Mém. Mus. Hist. Nat., Paris, 461 p.

Faroogi (S. I.), Subba Rao (B. R.) et Sharma (A. K.), 1965. - Studies on the parasites of Orthaga sp., a pest of Syzygium fruticosum Roxb. at Delhi. Beit. z. Ent., 15 (1-2), 179-198.

FORD (N.), 1922. - An undescribed Planidium of Perilampus from Conocephalus. Canad. Ent., 54, 199-204.

Howard (L. O.), 1891. - The biology of the Hymenopterous Insects of the Family Chalcididae. Proc. U.S. Nat. Mus., Washington, 14, 567-588.

LARIVIÈRE (M.) et ABonnenc (E.), 1958. - Sur un parasite de Phlebotomus freetownensis var. sudanicus Théodor, 1933 (Diptera, Psychodidae). Ann. Parasit. Hum. Comp., 33 (1-2), 112-114.

Martelli (G. H.), 1932. - Notizie biologiche et Morphologiche sull' Athalia colibri Christ. Lab. Zool. Genev. Agr. Portici Boll., 26, 313-333.

PARKer (H.-L.), 1924. - Recherches sur les formes post-embryonnaires des Chalcidiens. Ann. Soc. ent. Fr., 93, 261-379, 39 pl.

PhILIP (C. B.), 1949. - New North American Tabanidae (Diptera), parts I-III. Ann. ent. Soc. Amer., 42, 451-460.

PrincipI (M. M.), 1947. - Contributi allo studio dei Neurotteri italiani. V. Ricerche su Chrysopa formosa Brauer. Boll. Ist. ent. Bologna, 16, 134-175.

RAGEAU (J.), 1951. - Un cas de parasitisme accidentel par une larve primaire de Strepsiptère chez un Phlébotome. Ann. Parasit. Hum. Comp., 26 (5-6), 473-474, 2 phot.

Rondani (C.), 1872. - Degli Insetti parassiti e delle loro vittime. Boll. Soc. Entom. Ital., 4, 41-78.

—, 1876. - Repertorio degli Insetti parassiti e delle loro vittime. Ibid., 8, 237-258.

SMIRNOFF (W. A.), 1957. - Entomophaga, 2 (1).

SмITH (C. W.), 1939. - An exchange of grasshoper parasites between Argentina and Canada with notes on parasitism on native grasshoper. Seventieth Ann. Rep. Ent. Soc. Ont., 61-62. 
Sмith (H. S.), 1912. - IV. The Chalcidoid Genus Perilampus and its relations to the problem of parasite introduction. U.S. Dep. Agr., Bur. of Ent., Tech. Ser., 19 (4), 33-69.

SмITH (R. W.), 1958. - Parasites of nymphal and adults grasshopers (Orthoptera: Acrididae) in western Canada. Canad. Jour. Zool., 36, 217-262.

Thompson (W. R.), 1915. - Contribution à la connaissance de la larve Planidium (Hymenoptera, Chalcidoidea). Bull. Scient. France et Belgique, 48 (3), 319-349.

—, 1915. - Sur le cycle évolutif de Fortisia foeda. C.R. Soc. Biol., 78, 413.

TRIPP (H. A.), 1962. - The biology of Perilampus Say (Hymenoptera: Perilampidae), a Primary Parasite of Neodiprion swainei Midd. (Hymenoptera: Diprionidae) in Quebec, with Descriptions of the Egg and larval Stages. Canad. Ent., 94 (12), 1250-1270.

Wheeler (W. M.), 1907. - The Polymorphism of Ants with an Account of some singular Abnormalities due to Parasitism. Bull. Am. Mus. of Nat. Hist., 23, 2-21.

(Laboratoire de Zoologie et de Parasitologie,

Faculté de Médecine et de Pharmacie de Bordeaux).

\section{Note additionnelle}

Depuis l'envoi à la publication de notre note sur le parasitisme de Simulium damnosum par un planidium de Perilampidae, nous avons poursuivi nos recherches bibliographiques sur la question, en particulier en ce qui concerne le parasitisme d'insectes hématophages; ce qui nous amène, pour être complet, à ajouter les précisions suivantes à notre dernier chapitre :

Perilampidae D'insectes hématophages :

1. Chez les Glossines : Perilampus ruficornis F. $(=P$. violaceus $\mathrm{F}$.) a été rencontré au Nigéria chez Glossina morsitans Westwood (Ferrière, 1935 ; Thompson, 1943 ; Buxton, 1955 ; Jenkins, 1964).

2. Chez les Simuliidae : Contrairement à ce que nous avons écrit plus haut, des planidia de Perilampidae ont déjà été récoltés chez Simulium damnosum, mais leur description n'a pas été faite.

Lewis (1953) a récolté au Soudan 107 simulies parasitées par des planidia (2,6\% des 4.108 exemplaires examinés étaient parasités: 90 contenaient un planidium; 15 en contenaient deux et 2 en contenaient trois). Cet auteur donne un petit schéma du planidium en cause; ce dernier semble très proche (sinon identique) de celui que nous avons décrit. Lewis aboutit à la conclusion qu'il s'agit là d'un parasitisme accidentel.

Lewis (1960) trouve, au Sud Cameroun, 2,8 \% de S. damnosum infectées par des planidia; il cite d'autre part avoir rencontré le même type de parasite en Nigéria et en Sierra Leone.

3. Chez les Phlébotomes: Nous n'avons relevé que la référence de Larivière et Abonnenc (1958), déjà citée. 


\section{Chez les Tabanidae :}

Lavoipierre (1957) cite avoir récolté deux planidia, fixés à la face externe du labelle droit d'un exemplaire de Chrysops silacea Austen.

Nicholls (1920) (in Jenkins, 1964) cite des larves de Chalcidiens fixées à la trompe de Tahanidae et indique que des œufs de Tabanus sp. peuvent être parasités par un Chalcidien qui pourrait être un Perilampus.

\section{Bibliographie}

Buxton (P. A.), 1955. - The Natural History of tsetse flies. An Account of the Biology of the genus Glossina (Diptera). London School of Hyg. and trop. Med., Mem. $\mathrm{N}^{\circ}$ 10. H. K. Lewis \& $\mathrm{C}^{\circ}$, ed., London, 1 vol., 816 p., 47 pl., 165 fig.

FERRIère (C.), 1935. - Les Hyménoptères parasites des mouches tsétsé. Mitt. Schweiz. ent. Ges., 16, 328-340. (R.A.E., 1935, B, 23, 124).

JeNKINS (D. W.), 1964. - Pathogens, parasites and predators of medically important Arthropods. Annotated list and bibliography. Bull. O.M.S., 30 (suppl.), 150 p.

LAVoIPIERRE (M. M. J.), 1957. - Some ectoparasites of Chrysops silacea, the vector of Loa loa (Laboratory demonstration). Trans. R. Soc. trop. Med. Hyg., 51, 4.

LEwIS (D. J.), 1953. - Simulium damnosum and its relation to Onchocerciasis in the Anglo-Egyptian Sudan. Bull. Ent. Res., 43, 597-644.

Lewis (D. J.), 1960. - Observations on Simulium damnosum in the Southern Cameroons and Liberia. Ann. Trop. Med. Parasit., 54, (2), 208-223.

Nicholls (L.), 1920. - A Chalcid parasite of Diptera. Sci. and Ind. (Melb.), 2. 607-609. (R.A.E., 1920, B, 9, 45).

Thompson (W. R.), 1943. - A catalogue of the Parasites and Predators of Insects pests. Part 2: Parasites of the Dermaptera and Diptera. Belleville, Ont. Imp. Parasite Serv., 1 vol., 99 p. (R.A.E., 1944, B, 32, 67 et 144). 